\title{
Excrement Strikes Back: The Dark Side of Fecal Microbiota Transplantation
}

\author{
Madeleine Kleven \\ The Care Quality Research Group, Emeryville, CA, USA \\ Corresponding author: Madeleine Kleven, E-mail: madeleinekleven@gmail.com, ORCID: https://orcid.org/0000-0002-6913-9129
}

\section{Introduction}

Fecal microbiota transplantation (FMT, also known as bacteriotherapy), a somewhat obscure medical technique, has been historically utilized for hundreds of years throughout the world. Recently and astonishingly, modern medicine has begun to utilize FMT in the fight against Clostridium difficile infection (CDI) and rapidly increasing chronic gastrointestinal maladies such as Crohn's disease (CD), ulcerative colitis (UC), and Irritable bowel disease (IBD) [1,2]. The modus operandi of FMT is rather simple in that it transfers a healthy donor's stool into the recipient's gastrointestinal tract. That way, FMT can restore the patient's gut microbiome by enhancing the growth of non-pathogenic healthy bacteria in the place of detrimental commensal or antigenic organisms [3]. Thus far, the most commonly studied and documented use of FMT in North America is for Clostridium difficile and Clostridium difficile associated disease (CDAD).

CDAD causes severe colitis, which leads to significant morbidity and mortality worldwide and is often acquired by patients with depleted gut microbiota, either due to previous antibiotic use or other medical comorbidities [4]. CDI is one of the most common hospital-associated infections in developed countries, and with the emergence of hypervirulent strains resistant to fluoroquinolone antibiotics in both North America and Europe [5], Clostridium difficile has broadened to affect a growing number of community-based populations as well [6]. This is where
FMT comes into play: While antibiotic use in Clostridium difficile and the above conditions can further disrupt the microbiome, FMT aims to recolonize the gut flora with healthy microorganisms capable of re-establishing gut function and breaking the cycle of recurrent CDI and other dysbiotic bacteria $[7,8]$. The transplantation process often involves a colonoscopy, endoscopy, sigmoidoscopy, or enema and has proven to be an overall efficacious treatment.

\section{Risks Associated with FMT}

Fecal bacteriotherapy as a therapeutic intervention has immense benefits, but like many interventions, it presents some serious risks as well. In a systematic review conducted by Wang et al., adverse effects (AEs) related to fecal transplantation were identified and analyzed from a pool of 50 selected publications [9]. Because there is currently no standardized fecal transplantation method, the AEs related to the procedure were influenced by not only host factors, including recipient and donor health status but methods of administration and regimen of FMT. Wang et al. found that nearly $44 \%$ of patients who received upper gastrointestinal routes of FMT administration (e.g., nasogastric tube, nasojejunal tube, gastroscopy, nasoduodenal tube) were affected by FMT-attributable adverse effects, whereas only $17.7 \%$ of patients who underwent lower gastrointestinal routes (colonoscopy, sigmoidoscopy and retention enema) experienced adverse 
effects. Regardless of the administration method (upper or lower), the vast majority of adverse effects fell into the category of "abdominal discomfort." These included diarrhea, flatulence, bloating, cramps, transient fever, nausea, vomiting, constipation, and other generally mild, nonspecific symptoms [9]. In some rare instances, researchers reported adverse effects that were much more severe. These included cases of GI hemorrhage [10], appendicitis [11], UTI [12], norovirus [13], pneumonia [14], relapse in ulcerative colitis [15], and even death [16]. While these risks are not to be taken lightly, it is worth noting the difficulty in drawing a direct causal link to some of these adverse effects from FMT given the underlying immunocompromised status of the patients.

Whether from an existing infection, comorbidities, or chronic illness, many of the patients undergoing FMT have severe intestinal inflammation and an impaired mucosal barrier, making the transfer of novel microorganisms into the gastrointestinal tract a potentially risky procedure. When a physician introduces a plethora of foreign organisms into a patient's gut during FMT, this process can grant entry to a host of unwanted microbial guests. The greatest risk that FMT presents is the transmission of potentially lethal organisms directly into the digestive tract of transplant recipients and the resultant serious adverse reactions that may occur. Recently donor stool carrying extended-spectrum beta-lactamase (ESBL)-producing Escherichia coli was given to two immunocompromised adults receiving investigational fecal transplants. One of the individuals died from the resulting infection. In light of this tragedy, the US Food and Drug Administration (FDA) released an important safety alert on June $13^{\text {th }}, 2019$ warning about the potential dangers of FMT and outlining specific protective measures for FMT investigation [17]. With these new measures in place, FDA has halted many clinical trials involving FMT until investigators can demonstrate proper screening procedures to ensure donor stool is free of harmful organisms $[17,18]$. The medical community has yet to develop a standard screening test for donor stool; however, this action by the FDA has prompted many facilities to ramp up their screening processes.

\section{The Future}

FMT would most certainly benefit from more strict regulation and study; however, the procedure shows incredible potential. Genomic and phenotypic examination of the gut has shown researchers not only how the microbiome regulates physiological processes, but also how it initiates and predisposes humans and animals to disease [19]. The gastrointestinal tract is home to a diverse spectrum of microorganisms, of which strict anaerobic bacteria are the most abundant [20]. Because of their ability to interact with the intestinal lining, these bacteria have the ability to regulate intestinal permeability and the absorption, metabolism, and excretion of nutrients within the intestinal mucosa [21]. With these important regulatory functions, these bacteria have the ability to influence the body on a systemic rather than intestinally localized spectrum [22].

It has been established that dysbiosis of the microbiota, whether caused by an outside infiltrate such as Clostridium difficile or an aggressive commensal within the intestinal walls, leads to the pathogenesis of both intestinal and extraintestinal disorders [23]. Because of the diverse functionality of microbial organisms, researchers are recognizing the role of FMT in a broad spectrum of human diseases not only within the intestinal tract but in extra-intestinal organ systems as well. Fecal transplants and subsequent alteration of human flora have shown some success at remedying not only bacterial infections like Clostridium difficile, but also providing treatment options for autoimmune conditions such as Crohn's disease, irritable bowel disease, lupus, neurodevelopmental disorders, and allergic diseases [24]. The identification of commensal bacteria, which initiate specific lymphocytic responses, and the discovery of bacteria-derived proteins which act as superantigens, inducing nonspecific activation via molecular mimicry of self-reactive $\mathrm{B}$ and $\mathrm{T}$ cells, indicate a connection between the microbiome and autoimmunity [14].

As with any modern medical procedure, the safety and therapeutic efficacy must be well studied and evaluated. A clinical trial assessing the safety of FMT by enema be- 
gan this last January and is expected to examine the longterm benefits and side effects of this procedure [25]. As additional high-quality randomized control trials are conducted, we will be able to elucidate methods to improve FMT and safely utilize the procedure for a host of human diseases. Patients undergoing FMT should be aware of the potentially fatal risks associated with this procedure. However, they should be aware of the potential benefits as well. It is worthwhile considering undergoing FMT for some of the better tested gastrointestinal diseases, however at home, FMT procedures should be discouraged, and patients looking to have a transplant should always make sure donor stool is thoroughly tested for lethal pathogens.

\section{References}

1. Ghione S, Sarter H, Fumery M, Armengol-Debeir L, Savoye G, Ley D, et al.; Epimad Group. Dramatic increase in incidence of ulcerative colitis and Crohn's disease (19882011): a population-based study of French adolescents. Am J Gastroenterol 2018;113:265-72.

2. Sýkora J, Pomahačová R, Kreslová M, Cvalínová D, Štych P, Schwarz J. Current global trends in the incidence of pediatric-onset inflammatory bowel disease. World J Gastroenterol 2018;24:2741-63.

3. Karadsheh $\mathrm{Z}$ and Sule S. Fecal transplantation for the treatment of recurrent Clostridium difficile infection. $\mathrm{N}$ Am J Med Sci 2013;5:339-43.

4. Kyne L, Warny M, Qamar A, Kelly CP. Asymptomatic carriage of Clostridium difficile and serum levels of IgG antibody against toxin A. N Engl J Med 2000;342:390-7.

5. He M, Miyajima F, Roberts P, Ellison L, Pickard DJ, Martin MJ, et al. Emergence and global spread of epidemic healthcare-associated Clostridium difficile. Nat Genet 2013;45:109-13.

6. Burke KE and Lamont JT. Clostridium difficile infection: a worldwide disease. Gut Liver 2014;8:1-6.

7. Lewis BB, Buffie CG, Carter RA, Leiner I, Toussaint NC, Miller LC, et al. Loss of microbiota-mediated colonization resistance to Clostridium difficile infection with oral vancomycin compared with metronidazole. J Infect Dis 2015;212:1656-65.

8. Kassam Z, Hundal R, Marshall JK, Lee CH. Fecal transplant via retention enema for refractory or recurrent Clostridium difficile infection. Arch Intern Med 2012;172: 191-3.

9. Wang S, Xu M, Wang W, Cao X, Piao M, Khan S, et al. Systematic review: adverse events of fecal microbiota transplantation. PLoS One 2016;11:e0161174.
10. MacConnachie AA, Fox R, Kennedy DR, Seaton RA. Faecal transplant for recurrent Clostridium difficile-associated diarrhoea: a UK case series. QJM 2009;102:781-4.

11. Pierog A, Mencin A, Reilly NR. Fecal microbiota transplantation in children with recurrent Clostridium difficile infection. Pediatr Infect Dis J 2014;33:1198-200.

12. Silverman MS, Davis I, Pillai DR. Success of self-administered home fecal transplantation for chronic Clostridium difficile infection. Clin Gastroenterol Hepatol 2010;8: 471-3.

13. Schwartz M, Gluck M, Koon S. Norovirus gastroenteritis after fecal microbiota transplantation for treatment of Clostridium difficile infection despite asymptomatic donors and lack of sick contacts. Am J Gastroenterol 2013; 108:1367.

14. Kelly CR, Khoruts A, Staley C, Sadowsky MJ, Abd M, Alani M, et al. Effect of fecal microbiota transplantation on recurrence in multiply recurrent Clostridium difficile infection: a randomized trial. Ann Intern Med 2016;165: 609-16.

15. De Leon LM, Watson JB, Kelly CR. Transient flare of ulcerative colitis after fecal microbiota transplantation for recurrent Clostridium difficile infection. Clin Gastroenterol Hepatol 2013;11:1036-8.

16. Aas J, Gessert CE, Bakken JS. Recurrent Clostridium difficile colitis: case series involving 18 patients treated with donor stool administered via a nasogastric tube. Clin Infect Dis 2003;36:580-5.

17. Administration-USFDA. Important safety alert regarding use of fecal microbiota for transplantation and risk of serious adverse reactions due to transmission of multi-drug resistant organisms. U.S. Food \& Drug. https://www.fda. gov/vaccines-blood-biologics/safety-availability-biologics/important-safety-alert-regarding-use-fecal-microbiota-transplantation-and-risk-serious-adverse (Updated on 13 Jun 2019)

18. Grady D. Fecal transplant is linked to a Patient's death, the F.D.A. warns. The New York Times. https://www.nytimes. com/2019/06/13/health/fecal-transplant-fda.html (Updated on 13 Jun 2019)

19. Turnbaugh PJ, Ley RE, Hamady M, Fraser-Liggett CM, Knight R, Gordon JI. The human microbiome project. Nature 2007;449:804-10.

20. Dethlefsen L, McFall-Ngai M, Relman DA. An ecological and evolutionary perspective on human-microbe mutualism and disease. Nature 2007;449:811-8.

21. Tremaroli V and Bäckhed F. Functional interactions between the gut microbiota and host metabolism. Nature 2012;489:242-9.

22. Kau AL, Ahern PP, Griffin NW, Goodman AL, Gordon JI. Human nutrition, the gut microbiome and the immune system. Nature 2011;474:327-36.

23. Carding S, Verbeke K, Vipond DT, Corfe BM, Owen LJ. 
Dysbiosis of the gut microbiota in disease. Microb Ecol Health Dis 2015;26:26191.

24. Eiseman B, Silen W, Bascom GS, Kauvar AJ. Fecal enema as an adjunct in the treatment of pseudomembranous enterocolitis. Surgery 1958;44:854-9.
25. National Institute of Health. Clinical trial testing fecal microbiota transplant for recurrent diarrheal disease begins. https://www.nih.gov/news-events/news-releases/ clinical-trial-testing-fecal-microbiota-transplant-recurrent-diarrheal-disease-begins (Updated on 14 Jan 2019) 